\title{
Prognosis of primary ab externo surgery for primary congenital glaucoma
}

\author{
Thomas S Dietlein, Philipp C Jacobi, Günter K Krieglstein
}

\begin{abstract}
Background-The strategy of pressure reducing surgery in primary congenital glaucoma has changed over the last decade. Ab externo filtering procedures-for example, trabeculectomy or trabeculotomy combined with trabeculectomy, have now been accepted even as primary intervention.

Methods-The authors reviewed 61 eyes in 35 consecutive patients with primary congenital glaucoma, who underwent different types of initial ab externo surgery between 1988 and 1996 (median follow up 36 months) to determine the efficacy of different surgical techniques and the influence of various risk factors.

Results-Trabeculotomy was performed in 17 eyes $(27.9 \%)$, trabeculotomy with trabeculectomy in 15 eyes $(24.6 \%)$, and trabeculectomy in 29 eyes $(47.5 \%)$. Regarding age, preoperative intraocular pressure, corneal diameter, ocular axial length, and incidence of corneal haze the subgroups were comparable. Success rates of trabeculotomy, trabeculectomy, and a combined procedure did not significantly differ when assessed by life table analysis. Patient age under 3 months $(p=0.014)$ and an ocular axial length of 24 mm or more $(p=0.016)$ proved to be major risk factors for primary ab externo surgery failure. A second operation was necessary in 20 of 61 eyes $(32.8 \%)$ during follow up.

Conclusion-Prognosis of primary ab externo glaucoma surgery in primary congenital glaucoma seems to be governed more by the individual course and severity of the disease than by modification of surgical techniques.

(Br F Ophthalmol 1999;83:317-322)
\end{abstract}

Accepted for public

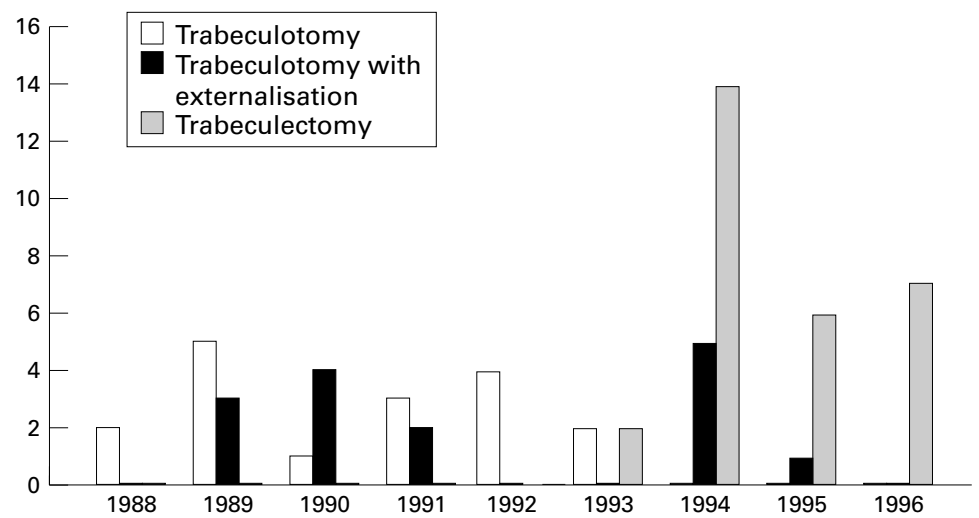

Figure 1 Graph demonstrating a change in surgical techniques of ab externo surgery performed in primary congenital glaucoma during the past decade in our department.
Strategies of antiglaucomatous surgery in primary congenital glaucoma have always been governed by historical trends. Goniotomy clinically introduced by Barkan in the 1940s was undoubtedly a great step forward in the surgical management of congenital glaucoma. ${ }^{12}$ However, good visibility of the chamber angle structures and considerable surgical experience are required for this delicate kind of ab interno surgery. Even if a good gonioscopic view is achieved by corneal epithelial removal, the handling with the goniotomy knife carries particular risks (for example, inadvertent damage to the lens and cyclodialysis) in the intraoperatively flattened anterior chamber of newborns. In the 1970s and 1980s trabeculotomy became an established alternative ab externo procedure in the surgical treatment of congenital glaucoma. ${ }^{3-7}$ This method of pressure reducing surgery does not depend on the visualisation of the chamber angle structures through an often cloudy cornea. However, an abnormally stretched anatomy of the limbus in congenital glaucoma frequently makes it difficult to clearly identify the lumen of Schlemm's canal that has to be cannulated for the trabeculotomy. Trabeculotomy can also be modified to a filtering procedure by excising a block of scleral tissue as in routine trabeculectomy. This trabeculotomy combined with trabeculectomy has been performed as a planned procedure in developmental glaucoma by different surgeons with reasonable results. ${ }^{8-10}$ Finally, in the late 1980 s and in the 1990s much better results for trabeculectomy in cases of primary congenital glaucoma were presented than those published in the decades before. ${ }^{11}{ }^{12}$ Therefore, filtering procedures once again became a promising alternative for surgical management of congenital glaucoma and today are generally accepted even as primary surgical intervention. ${ }^{13}{ }^{14}$ The fact that most ophthalmic surgeons are relatively familiar with this technique is a further reason for extending the application of trabeculectomy to primary surgery for congenital glaucoma.

The aim of our study was to investigate the outcome of trabeculotomy, trabeculectomy, and a combined procedure as initial surgical treatment in primary congenital glaucoma over the past decade in patients treated in our department.

Patients and methods

The operating room records of the Department of Ophthalmology, University of Cologne, were used to identify all consecutive patients undergoing primary pressure reducing 
Table 1 Age at glaucoma surgery and follow up depending on type of surgery

\begin{tabular}{llll}
\hline & $\begin{array}{l}\text { Eyes } \\
\text { (patients) }\end{array}$ & $\begin{array}{l}\text { Median age } \\
\text { (range) in } \\
\text { months }\end{array}$ & $\begin{array}{l}\text { Median } \\
\text { follow up } \\
\text { (range) in } \\
\text { months }\end{array}$ \\
\hline $\begin{array}{l}\text { Trabeculotomy } \\
\text { Trabeculotomy } \\
\text { combined with } \\
\text { trabeculectomy }\end{array}$ & $17(11)$ & $3(0-50)$ & $52(31-105)$ \\
$\begin{array}{c}\text { Trabeculectomy } \\
\text { Traber }\end{array}$ & $29(17)$ & $5(0-8)$ & $46(17-96)$ \\
\hline
\end{tabular}

surgery for primary congenital glaucoma from the middle of 1988 to 1996 ; patients with a history of previous surgery, with complex ocular syndromes (for example, Axenfeld-Rieger syndrome, aniridia, Sturge-Weber syndrome) or with any suspicion of secondary glaucoma were excluded.

Diagnosis of primary congenital glaucoma was always established by an examination under general anaesthesia using halothane. Informed consent for pressure reducing surgery was obtained from the parents at least 1 day before examination under anaesthesia.

Indication for pressure reducing surgery was established if four or more of the following criteria were fulfilled: (1) typical symptoms (epiphora, photophobia, blepharospasm), (2) cloudy cornea, (3) increased intraocular pressure $(>18 \mathrm{~mm} \mathrm{Hg}$ by applanation or $>23 \mathrm{~mm}$ $\mathrm{Hg}$ by indentation under deep general anaesthesia with halothane), (4) abnormally increased corneal diameter or ocular axial length in comparison with the growth curve of normal eyes, (5) if visible, a pathological excavation of the optic disc and dysgenetic signs of the chamber angle-for example, mesodermal remnants or fine iris roots, (6) primary congenital glaucoma of the contralateral eye.

Success of pressure reducing surgery was determined by an intraocular pressure $<18$ $\mathrm{mm} \mathrm{Hg}$ under general anaesthesia or $<21 \mathrm{~mm}$ $\mathrm{Hg}$ in the awake child measured by applanation, stable axial length, improvement or at least stability of the optic disc excavation, and clearing of the cornea. Our definition of success excluded any antiglaucomatous medical treatment. Visual function was not taken as a criterion, since the mean age of patients included in the study was too young to obtain reliable results concerning visual acuity or perimetry.

Choice of surgical approach depended on two particular factors: (1) the year in which surgery was performed (Fig 1), as primary trabeculectomy alone was not introduced until 1993, while trabeculotomy was the standard procedure in the late 1980s; (2) the intraopera- tive identification of Schlemm's canal: in at least four eyes an intended trabeculotomy or combined procedure turned into a trabeculectomy because Schlemm's canal could not be localised. Pressure reducing interventions were performed by different surgeons all experienced in the field of paediatric eye surgery under supervision of the same senior surgeon (GKK). All initial pressure reducing ab externo interventions took place under general anaesthesia. A limbus based conjunctival flap was dissected in one of the upper quadrants without excision of Tenon's capsule. Subsequently, a lamellar rectangular scleral flap of $4 \times 4 \mathrm{~mm}$ size was dissected crossing the grey-white borderline zone into the clear cornea. In the case of trabeculotomy a radial incision was done to find Schlemm's canal. A McPherson trabeculotome was inserted into the lumen of Schlemm's canal on either side of the incision, if possible, and carefully rotated into the anterior chamber with the other parallel arm as a guide. In the case of trabeculectomy, after reaching the grey-white border and limbal clear cornea a trabeculectomy piece of $1 \times 3 \mathrm{~mm}$ size was excised with the diamond knife and a small peripheral iridectomy was performed. In the case of trabeculotomy with trabeculectomy, both procedures were conducted subsequently as described. Finally, the scleral flap was loosely reaffixed using two 10-0 nylon sutures. The conjunctiva was closed with a running 8-0 Vicryl suture. The anterior chamber was filled with a balanced salt solution and the blood was washed out. Surgery was completed by a subconjunctival injection of dexamethasone and mezlocillin, and the eye was dressed with atropine and antibiotic corticosteroid ointment. Postoperative treatment comprised topical administration of combined antibiotic corticosteroid medication for 7-10 days.

Follow up data were collected from patients' charts, by direct contact with the external ophthalmologist and by voluntary re-examination in our department.

Statistical analysis was performed using the software program PRISM Version 2.0 (GraphPad Software, Inc, USA). For evaluation of the postoperative outcome, Kaplan-Meier survival curves for censored data were plotted, and significance levels determined using the log rank test. Differences of distribution for different risk factors between the subgroups treated by different $a b$ externo techniques were assessed by means of the Kruskal-Wallis test.

Table 2 Distribution of risk factors among the subgroups before primary surgery

\begin{tabular}{|c|c|c|c|c|c|c|c|c|c|}
\hline & \multicolumn{2}{|c|}{ Trabeculotomy } & \multicolumn{2}{|c|}{ Combined procedure } & \multicolumn{2}{|c|}{ Trabeculectomy } & \multicolumn{2}{|l|}{ All groups } & \multirow[b]{2}{*}{$\begin{array}{l}\text { Kruskal-Walli } \\
\text { test ( } p \text { value) }\end{array}$} \\
\hline & $\begin{array}{l}\text { Mean value } \\
(S D)\end{array}$ & No & $\begin{array}{l}\text { Mean value } \\
(S D)\end{array}$ & No & $\begin{array}{l}\text { Mean value } \\
(S D)\end{array}$ & No & $\begin{array}{l}\text { Mean value } \\
(S D)\end{array}$ & No & \\
\hline Perkins tonometry $(\mathrm{mm} \mathrm{Hg})$ & $20.1(5.9)$ & 17 & $16.4(5.9)$ & 13 & $17.4(9.1)$ & 26 & $18.0(7.6)$ & 56 & 0.22 \\
\hline Schiotz tonometry (mm Hg) & $24.5(7.2)$ & 17 & $21.2(7.5)$ & 13 & $22.1(6.6)$ & 27 & $22.6(6.9)$ & 57 & 0.53 \\
\hline Axial length (mm) & $23.0(3.0)$ & 17 & $21.9(1.7)$ & 15 & $22.1(3.3)$ & 27 & $22.3(2.9)$ & 59 & 0.37 \\
\hline Corneal diameter (mm) & $13.3(0.9)$ & 16 & $13.2(1.0)$ & 11 & $13.2(2.0)$ & 25 & $13.2(1.5)$ & 52 & 0.98 \\
\hline Cup-disc ratio & $0.7(0.28)$ & 5 & $0.7(0.18)$ & 10 & $0.7(0.25)$ & 13 & $0.7(0.22)$ & 28 & 0.87 \\
\hline Corneal haze & $88.23 \%$ & 17 & $73.33 \%$ & 15 & $75.86 \%$ & 29 & $78.68 \%$ & 61 & 0.59 \\
\hline
\end{tabular}


- Trabeculotomy

$\Delta$ Combined trabeculotomy-trabeculectomy - Trabeculectomy

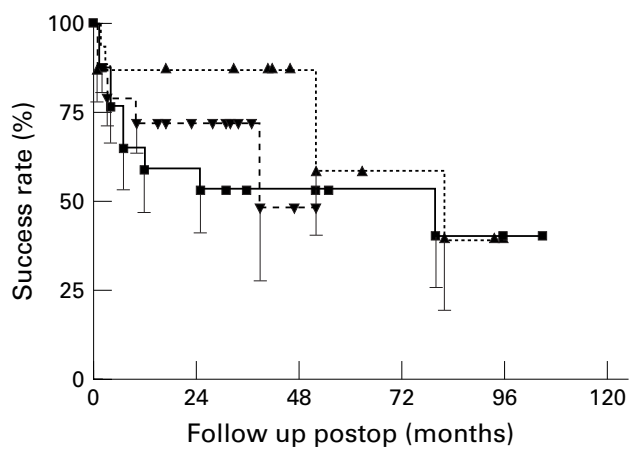

Figure 2 Censored subjects appear as data points in the flat part of the survival curve, the error bars (below) show the standard error of survival. Kaplan-Meier curve of censored survival data shows no significant difference in outcome between trabeculotomy, trabeculectomy, and combined procedure.

\section{Results}

SURGICAL OUTCOME

Primary pressure reducing surgery in patients with newly diagnosed primary congenital glaucoma was performed in 61 eyes (32 right eyes, 29 left eyes) of 35 patients. Among the 35 patients with primary congenital glaucoma 21 $(60 \%)$ were male and $14(40 \%)$ were female. Unilateral glaucoma was found in nine patients $(25.7 \%)$, and bilateral glaucoma in 26 (74.3\%).

Primary trabeculotomy was performed in 17 eyes $(27.9 \%)$ of 11 patients, primary trabeculectomy in 29 eyes $(47.5 \%)$ of 17 patients, and primary combined trabeculotomytrabeculectomy in 15 eyes $(24.6 \%)$ of 10 patients. In 22 patients with bilateral glaucoma the same type of surgery was conducted in both eyes. Only in four patients were different techniques of surgery performed in the two eyes (combined procedure and trabeculectomy or trabeculotomy). Age at initial surgery and follow up with range of values are indicated in Table 1 . The difference regarding age at initial surgery was not significant $(p=0.54)$, while in follow up of the subgroups clear differences were found $(\mathrm{p}<0.001)$ owing to the fact that trabeculectomy only became a primary procedure in recent years (1993-6). Distribution of various clinical factors were similar for all surgical subgroups assessed by the Kruskal-Wallis test as indicated in Table 2.

Kaplan-Meier survival analysis (Fig 2) revealed no significant difference in the surgical outcome between trabeculotomy and trabeculectomy (log rank test; $\mathrm{p}=0.5)$, nor between the combined procedure and trabeculotomy (log rank test; $\mathrm{p}=0.37$ ) or trabeculectomy (log rank test; $\mathrm{p}=0.25$ ). Success rates after 6, 24,

Table 3 Success rates of trabeculotomy, trabeculectomy, and a combined procedure

\begin{tabular}{lllllllll}
\hline & \multicolumn{2}{l}{ Trabeculotomy } & & \multicolumn{2}{c}{ Combined procedure } & & \multicolumn{2}{c}{ Trabeculectomy } \\
\cline { 2 - 3 } Postoperative follow up & Success rate & SEM & & Success rate & SEM & & Success rate & SEM \\
\hline 6 months & 0.77 & 0.1 & & 0.87 & 0.09 & & 0.79 & 0.08 \\
24 months & 0.59 & 0.12 & & 0.87 & 0.09 & & 0.72 & 0.09 \\
60 months & 0.53 & 0.12 & & 0.58 & 0.18 & & 0.48 & 0.2 \\
\hline
\end{tabular}

and 60 months are indicated in Table 3. During follow up 20 eyes $(32.8 \%)$ of 14 patients required secondary pressure reducing surgery. Combined trabeculectomytrabeculotomy was performed in one eye, the remaining cases were treated by trabeculectomy as secondary intervention. Secondary filtering procedures (Fig 3) showed a significantly higher failure rate than primary trabeculectomy $(\mathrm{p}=0.03)$.

Taking into consideration large axial length and corneal diameters, high intraocular pressure, and young age at time of surgery as independent risk factors for surgical failure, we studied the outcome in patients with a specific risk factor by life table analysis. Buphthalmic eyes in patients younger than 3 months $(n=20$, $\mathrm{p}=0.014)$ and with an axial length of $>24 \mathrm{~mm}$ $(\mathrm{n}=12, \mathrm{p}=0.016)$ actually had a significantly different outcome from the remaining eyes (Figs 4 and 5), while the outcome in patients with corneal diameter of $>14 \mathrm{~mm}(\mathrm{n}=14$, $\mathrm{p}=0.44$ ) and a preoperative intraocular pressure of $>24 \mathrm{~mm} \mathrm{Hg}$ under general anaesthesia $(\mathrm{n}=13, \mathrm{p}=0.41)$ was not significantly different. Outcome in Turkish or Arabian patients with glaucomatous eyes who underwent pressure reducing surgery was not significantly worse $(n=10 ; p=0.165)$ than that in white patients.

\section{COMPLICATIONS}

Chamber angle bleeding with small hyphaema occurred in 11 eyes (65\%) during trabeculotomy, in four eyes $(27 \%)$ during combined trabeculotomy-trabeculectomy, and in seven eyes $(24 \%)$ during primary trabeculectomy. The blood was usually absorbed within the first postoperative day. Prolapse of ciliary processes was reported for two eyes during trabeculectomy $(7 \%)$ and for one eye during the combined procedure $(6 \%)$. Postoperative iris incarceration was reported in one eye following trabeculotomy with trabeculectomy $(6 \%)$ and in three eyes after trabeculectomy $(11 \%)$, but pressure reducing surgery was successful in each of the four eyes.

Severe postoperative complications (within 6 months after surgery) comprised subluxation of the crystalline lens in one eye following pri-

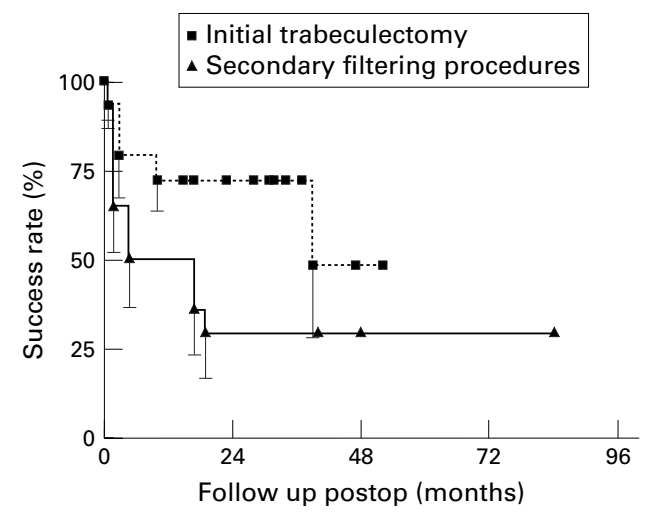

Figure 3 Censored subjects appear as data points in the flat part of the survival curve, the error bars (below) show the standard error of survival. Kaplan-Meier curve of censored survival data of primary trabeculectomy differs significantly from secondary filtering interventions $(p=0.02)$. 


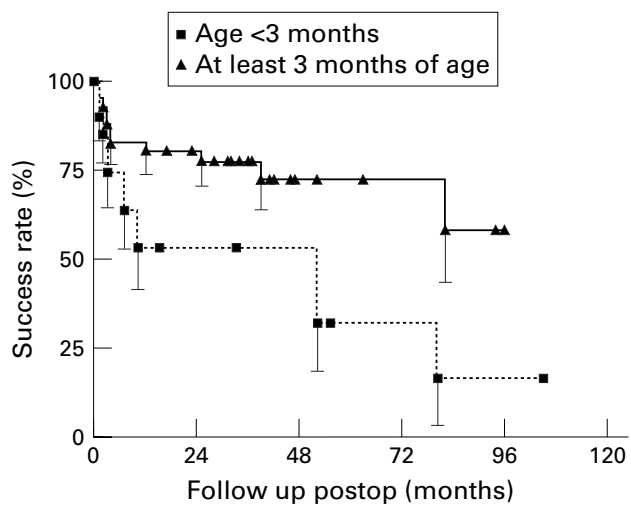

Figure 4 Censored subjects appear as data points in the flat part of the survival curve, the error bars (below) show the standard error of survival. Ab externo surgery in patients aged younger than 3 months show poorer outcome than in older patients $(p=0.014)$.

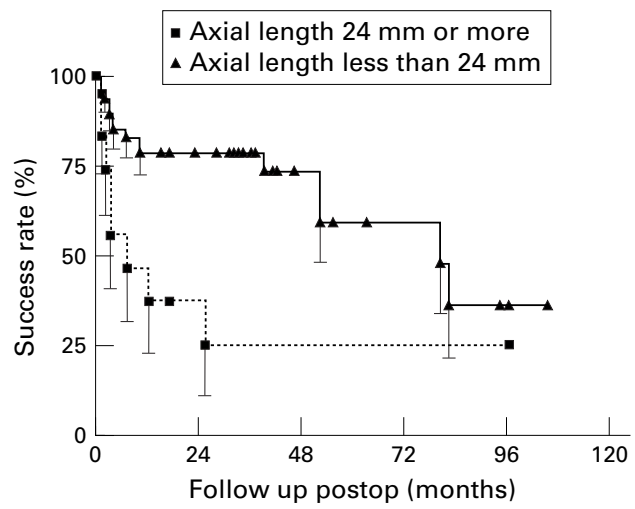

Figure 5 Censored subjects appear as data points in the flat part of the survival curve, the error bars (below) show the standard error of survival. Ab externo surgery of eyes with myopic axial length likewise show significantly $(p=0.016)$ worse results than eyes with axial length $<24$

mm.

mary trabeculectomy and cataract formation in another eye following secondary trabeculectomy. Long standing ocular hypotony or severe choroidal effusion was only observed in one eye in which a giant tear with retinal detachment was discovered by examination under general anaesthesia. Retinal detachment occurred in two eyes-in one case following primary combined procedure, in another following secondary trabeculectomy. Both these eyes received vitreoretinal surgery to reattach the retina using silicone oil. In one of the eyes further retinal detachment was observed following removal of silicone oil requiring further vitreoretinal surgery. Despite silicone oil treatment, both eyes developed ocular hypotony with clinical signs of phthisis.

\section{Discussion}

Retrospective studies comparing the outcome of different surgical procedures have to be evaluated with caution, especially if the decision to operate in a certain way follows an obvious historical trend as it does here. Moreover, the small number of patients leads to a limited statistical power of the study. On the other hand, primary congenital glaucoma represents an extraordinarily rare disease and the psychological situation of initial diagnosis makes it very difficult to convince parents to include their diseased child in a randomised prospective study. Since no significant differences were found in the distribution of well known risk factors among the subgroups treated by trabeculotomy, trabeculectomy, or the combined procedure we were able to attempt a statistical comparison of outcome between these surgical subgroups.

Criteria for surgical success in the treatment of congenital glaucoma mostly comprise resolution of the corneal oedema, stabilisation of the horizontal corneal diameters, axial length, and the cup-disc ratio, while tolerable intraocular pressure varies from 14 up to $22 \mathrm{~mm}$ $\mathrm{Hg}^{45111215}$ Prognosis of any surgery in primary congenital glaucoma is strongly influenced by the individual risk factors of the patients included into study. A trabeculectomy study performed by Fulcher and coworkers ${ }^{13}$ included no patients with congenital glaucoma younger than 3 months and no patients who had undergone previous surgery, while in Beauchamps and Parks' study ${ }^{16}$ proclaiming poor prognosis for trabeculectomy in congenital glaucoma, most patients were older than 4 years and had already undergone multiple operations. In our study 25 eyes (41\%) showed clinical manifestation and underwent primary glaucoma surgery within the first 3 months after birth. This has to be kept in mind when comparing our success rates after 2 and 5 years with other published studies.

Depending on follow up and risk profile in the study patients the success rates published in the literature for primary trabeculotomy and for trabeculectomy in congenital glaucoma range between $50 \%$ and more than $90 \%{ }^{10-12}{ }^{15}{ }^{17-19}$ This is in line with results published earlier for goniotomy. ${ }^{15} 2021$ Debnath et $a l,{ }^{17}$ who clearly described the distribution of risk factors in their study group, found a 1 year success rate of $54 \%$ for trabeculectomy compared with $67 \%$ for trabeculotomy. However, this difference was not significant. The authors concluded that their high failure rate might be influenced by an ethnic factor which is well known for scarring after trabeculectomy in non-white people. Besides, the portion of patients manifesting glaucoma soon after birth was relatively high in this study and obviously predisposed to poor results for both procedures in general. Elder, who exclusively studied Palestinian patients younger than 1 year, reported better results for combined trabeculotomy-trabeculectomy procedures $(93.5 \%)$ after 2 years compared with trabeculectomy ${ }^{10}$; however, the incidence of corneal haze in the trabeculectomy group was obviously higher $(82 \%$ versus $56 \%$ ), whereas other preoperative factors (for example, age, intraocular pressure, corneal diameters) were similar in both groups. The author explained the superior outcome of the combined procedure by improving outflow through the trabecular meshwork as well as through the sclera. Although combined procedures likewise seemed to have a favourable outcome after 2 years in our study, the advantage of this procedure over trabeculectomy was far from statisti- 
cally significant according to the life table analysis in our small patient group. Beauchamps and Parks, who mainly described secondary trabeculectomies, did not recommend this procedure, as they saw a failure rate of $40 \%$ in 10 buphthalmic eyes, associated with severe intra- and postoperative complications such as vitreous loss, endophthalmitis, retinal detachment, and scleral collapse. ${ }^{16}$ Vitreous prolapse with subluxation of the lens and uveitis as possible risks of trabeculectomy were also indicated by Rao et al, ${ }^{19}$ while shallow anterior chamber and hyphaema as the most common postoperative complications always resolved spontaneously. In contrast with these studies other groups reported much better results several years after primary trabeculectomy for congenital glaucoma. Burke and Bowell reported a surgical success in 18 of 21 eyes $(86 \%)$ with congenital glaucoma after a mean follow up of nearly 4 years ${ }^{11}$; in the same patients group Fulcher et al even observed a 5 year success rate of $92 \%$ after the first trabeculectomy in 13 eyes with primary congenital glaucoma, although no patients younger than 4 months of life, nor patients with a corneal diameter larger than $13.5 \mathrm{~mm}$ were treated in this study. ${ }^{13}$ The rate of re-surgery $(34 \%)$ after initial trabeculectomy in the study of Detry-Morel and Feron was similar to ours; they also underlined poor prognosis for neonatal forms of congenital glaucoma and highly myopic eyes. ${ }^{12}$

Although primary congenital glaucoma is described as an entity with the leading pathological feature of trabeculodysgenesis resulting in pathologically increased outflow obstruction, ${ }^{22}{ }^{23}$ the starting point for pressure reducing surgery varies considerably and the prognosis of any surgery is thought to be influenced by the individual nature of dysgenesis. ${ }^{5141524}$ Pronounced symptoms of high intraocular pressure during the first days of life in a neonate are a limiting factor for the outcome of pressure reducing surgery in congenital glaucoma. ${ }^{512} 1425$ Russell-Eggitt et al also reported a bad outcome for goniotomy in very young patients with congenital glaucoma, thereby confirming the importance of age for individual prognosis whatever the pressure reducing surgery. ${ }^{21}$

Any association with other ocular or systemic abnormalities evidently worsens surgical prognosis in congenital glaucoma. ${ }^{15} 24$ Axial length of the eye is also a critical factor, whereas corneal diameter was not found to influence outcome in our study. Possibly, because of the stretched limbal anatomy, the reading of the corneal diameter by calipers is not as precise as A-scan measurement of the axial length. Lack of prognostic power for the preoperative intraocular pressure should mainly be attributed to measurement in deep general anaesthesia. ${ }^{26} \mathrm{~A}$ massive decrease of intraocular pressure (IOP) has been shown in animal models after application of halothane. ${ }^{27}$ Consequently, it is important to consider that normal intraocular pressure in children ranges between 9 and $12 \mathrm{~mm} \mathrm{Hg}$ under general anaesthesia with halothane. IOP measure- ments between 16 and $18 \mathrm{~mm} \mathrm{Hg}$ might already be an indication of glaucoma when using halothane. Ketamine anaesthesia avoids this considerable decrease of IOP, but can lead transiently to a minor increase of $\mathrm{IOP}^{28}$ Ketamine anaesthesia in young children was abandoned in our department of anaesthesiology owing to the relatively long lasting effects of ketamine and the safety of short anaesthesia with volatile halothane.

Although trabeculectomy is widely performed by many ophthalmic surgeons, this procedure remains technically difficult in cases of congenital glaucoma as the limbal anatomy is usually distorted and the sclera extraordinarily thin. ${ }^{14}$ This can lead to inadvertent scleral perforation during preparation of the scleral flap. Another potential risk can be the prolapse of vitreous or ciliary body processes through the peripheral iridectomy into the trabeculectomy opening, especially with a primarily dislocated lens. Retinal detachment after filtering surgery in two patients of our group clearly demonstrated the high susceptibility to retinal tears of the stretched buphthalmic eye. Rice also reported several cases of retinal detachment in his large series of 246 eyes with congenital glaucoma after goniotomy, ${ }^{20}$ although the postoperative risk for retinal tears should be higher in filtering procedures owing to frequent postoperative hypotony of the eye. Considering clinical results after goniotomy it seems reasonable to perform goniotomy or similar pressure reducing ab interno surgery (for example, endoscopically guided goniotomy or laser goniotomy $)^{29} 30$ as a primary intervention, because the conjunctiva is not damaged and thus future surgery is not prejudiced. In order to avoid the use of antimetabolites for as long as possible, a surgical strategy sparing the conjunctiva from scarring at an early stage would be desirable in primary congenital glaucoma, since future re-surgery has always to be taken into account. ${ }^{31}$ Application of mitomycin or fluorouracil in young children with a long life expectancy is associated with the risk of late toxicity and potential mutageneity of these antimetabolites, even though the filtering procedures combined with the use of mitomycin have become more and more routine in antiglaucomatous surgery. ${ }^{32-34} \mathrm{Be}-$ sides, rupture of thin walled blebs, wound leakage, late endophthalmitis, and long standing hypotony might be further deleterious complications of filtering procedures with mitomycin. ${ }^{35-37}$ However, in refractory cases the use of antimetabolites or other methods ${ }^{38}$ to prevent scarring is unavoidable where goniosurgery and $a b$ externo procedures without antimetabolites have failed.

We conclude that the different options for $a b$ externo glaucoma surgery do not clearly influence the outcome of surgery in primary congenital glaucoma. Moreover, early manifestation and large ocular dimensions are the key to a limited prognosis of any pressure reducing surgery in primary congenital glaucoma. As re-surgery is often inevitable in congenital glaucoma owing to long life expectancy, a stepwise surgical strategy has to be devised, 
starting with ab interno surgery and proceeding to conventional ab externo procedures, before using antimetabolites and cyclodestructive methods in refractory cases.

1 Barkan O. Technique of goniotomy. Arch Ophthalmol 1938;19:217-21.

2 Barkan O. Surgery of congenital glaucoma. Review of 196 eyes operated by goniotomy. Am $\mathcal{F}$ Ophthalmol 1953;36: 1523-34.

3 Harms H, Dannheim R. Epicritical consideration of 300 cases of trabeculotomy ab externo. Trans Ophthalmol Soc UK 1970;89:491-9.

4 Luntz MH, Livingstone DG. Trabeculotomy ab externo and trabeculectomy in congenital and adult onset glaucoma. Am f Ophthalmol 1977;83:174-9.

5 Quigley HA. Childhood glaucoma: results with trabeculotomy and study of reversible cupping. Ophthalmology 1982;89:219-26.

6 Anderson DR. Trabeculotomy compared to goniotomy for glaucoma in children. Ophthalmology 1983;90:805-6.

7 Grehn F. The value of trabeculotomy in glaucoma surgery. Curr Opin Ophthalmol 1995;6:52-60.

8 Board RJ, Shields MB. Combined trabeculotomytrabeculectomy for the management of glaucoma associated with Sturge-Weber syndrome. Ophthalmic Surg 1981, 12:813-7.

9 Agarwal HC, Sandramouli S, Sihota R, et al. Sturge-Weber syndrome: management of glaucoma with combined trabeculotomy and trabeculectomy. Ophthalmic Surg 1993; 24:399-402.

10 Elder MJ. Combined trabeculotomy-trabeculectomy compared with primary trabeculectomy for congenital glaucoma. Br f Ophthalmol 1994;78:745-8.

11 Burke JP, Bowell R. Primary trabeculectomy in congenital glaucoma. Br f Ophthalmol 1989;73:186-90.

12 Detry-Morel M, Feron EM. Trabeculectomy in congenital glaucoma: retrospective medium and long term results. Bull Soc Belge Ophtalmol 1996;262:143-51.

13 Fulcher T, Chan J, Lanigan B, et al. Long term follow up of primary trabeculectomy for infantile glaucoma. $\mathrm{Br} \mathscr{f}$ primary trabeculectomy for
Ophthalmol 1996;80:499-502.

14 Khaw PT. What is the best primary surgical treatment for the infantile glaucomas? (Editorial). Br f Ophthalmol 1996; 80:495-6.

15 DeLuise VP, Anderson DR. Primary infantile glaucoma. Surv Ophthalmol 1983;28:1-19.

16 Beauchamps GR, Parks MM. Filtering surgery in children barriers to success. Ophthalmology 1979;86:170-80.

17 Debnath SC, Teichmann KD, Salamah K. Trabeculectomy versus trabeculotomy in congenital glaucoma. $\mathrm{Br} \mathcal{F}$ Ophthalmol 1989;73:608-11.

18 Elder MJ. Congenital glaucoma in the West Bank and Gaza strip. Br f Ophthalmol 1993;77:413-16.
19 Rao KV, Sai CM, Babu BV. Trabeculectomy in congenital glaucoma. Indian $\mathcal{f}$ Ophthalmol 1984;32:439-40.

20 Rice NSC. The surgical management of the congenital glaucomas. Aust $\mathcal{F}$ Ophthalmol 1977;5:174-9.

21 Russell-Eggitt IM, Rice NSC, Jay B, et al. Relapse following goniotomy for congenital glaucoma due to trabecular dysgenesis. Eye 1992;6:197-200

22 Hoskins DH, Shaffer RN, Hetherington J. Anatomical classification of the developmental glaucomas. Arch Ophhalmol 1984;104:1331-6.

23 Barsoum-Homsy M, Chevrette L. Incidence and prognosis of childhood glaucoma. Ophthalmology 1986;93:1323-7.

24 Wallace DK, Plager DA, Snyder SK, et al. Surgical results of secondary glaucomas in childhood. Ophthalmology 1998; 105:101-11.

25 Haas J. Principles and problems of therapy in congenital glaucoma. Invest Ophthalmol 1964;7:140-6.

26 Ausinsch B, Munson ES, Levy NS. Intraocular pressure in children with glaucoma during halothane anesthesia. Ann Ophthalmol 1978;9:1391-4.

27 Krupin T, Feitl M, Roshe R, et al. Halothane anesthesia and aqueous humor dynamics in laboratory animals. Invest Ophthalmol 1980;19:518-21.

28 Jantzen JPAH. Anesthesia and intraocular pressure. Anaesthesist 1988;37:458-69.

29 Joos KM, Wallace LM, Folberg R. Experimental endoscopic goniotomy. Ophthalmology 1993;100:1066-70.

30 Jacobi PC, Dietlein TS, Krieglstein GK. Experimental microendoscopic photoablative laser goniotomy as a surgi$\mathrm{cal}$ model for the treatment of dysgenetic glaucoma. Graefes Arch Clin Exp Ophthalmol 1996;234:670-6.

31 Russell-Eggitt IM. In defence of goniotomy (letter). $\mathrm{Br} F$ Ophthalmol 1995;79:709.

32 Susann R Jr, Oltragge EW, Carani JCE, et al. Mitomycin as adjunct chemotherapy with trabeculectomy in congenital and developmental glaucomas. F Glaucoma 1995;4:151-7.

33 Agarwal HC, Sood NN, Sihota R, et al. Mitomycin-C in congenital glaucoma. Ophthalmic Surg Lasers 1997;28:97985 .

34 Mandal AK, Walton DS, John $\mathrm{T}$, et al. Mitomycin-C augmented trabeculectomy in refractory congenital glaucoma. Ophthalmology 1997;104:996-1001.

35 Greenfield DS, Parrish II RK. Bleb rupture following filtering surgery with mitomycin-C. Clinicopathological correlations. Ophthalmic Surg Lasers 1996;27:876-7.

36 Greenfield DS, Suner IJ, Miller MP, et al. Endophthalmiti after filtering surgery with mitomycin. Arch Ophthalmol 1996;114:943-9.

37 Zacharia PT, Deppermann SR, Schuman JS. Ocular hypotony after trabeculectomy with mitomycin-C. Am f Ophthalmol 1993;116:314-26.

38 Miller MH, Rice NS. Trabeculectomy combined with beta irradiation for congenital glaucoma. Br f Ophthalmol 1991; 75:584-90 